\title{
Efecto de la Hidratación Oral y Realimentación Precoz en la Evolución de la Diarrea Aguda del Lactante
}

\author{
Nutr. Paulina Cordero T. ${ }^{1 *}$; Dra. Magdalena Araya Q. 1 ; Dr. Julio Fispinoza M. ${ }^{1}$; \\ T.M. Guillermo Figucroa G. ${ }^{2}$; T.M. Isolda Pacheco M.' ; Dr. Oscar Brunser T.1. \\ Oral Rehydration and Early Refeeding in Infants \\ with Acute Diarrhoea
}

\begin{abstract}
A follow-up of three groups of outpatients under 2 ycars of age $(N=15$ each), who consulted for acute diarshea was carried during 35 days. In agreement with current therapeutic schemes used at some Health Centers if Santiago, Group 1 patients were sehydrated by the oral route with sugar-added water and cautiously refed, so that, the child's food intake prior to the episode could be attained again within one week. These children also received Furazolidone orally. Group 2 patients were rehydrated orally with a commercial solution and refed as to reach their previuos food intake within 3 days. In Group 3, patients were rehydrated orally with a solution prepared by mothers at home, following WHO recommendations; they also attained thei previous intakes within three days. Children of Groups 2 and 3 did not receive antibiotics. During the episode and until day 35, whildren in Groups 2 and 3 showed significantly greater mean daily weight increments $(p<0.001)$. All cases had benign clinical courses, none of them required $i, v$. fluids or hospitalization. Treatment provided to Group 1 succeeded in replacing the fluids lost duc to the diarrheal episode and avoided further detcrioration of nutritional status. Schemes providing oral rehydration solutions with adecuate amounts of sodjum, potassium and glucose followed by carly tefeeding result in greater improventents of nutritional anthropometric parameters and this is obtained in a shorter period of tine.
\end{abstract}

(Key words: Acute diarhoea, Early refeeding. Oral rehydration. Weight gain).

La diarrea aguda es la principal calša de morbilidad y mortalidad en paises en desarrollo $y$

1. Unidad de Gastroenterología, INTA.

2. Unidad de Microbiología, INTA

* Este trabajo formó parto de la Tesis para obtener el grado de Magister en Nutrición Humana, INTA. Universidad de Chile. constituye uno de los factores más importantes entre los que condicionan la aparición de desnutrición infantil en ellos ${ }^{1-3}$.

El tratamiento de la diarrea aguda tiene por propósito recuperar el contenido de agua y electrolitos, mantener el estado nutricional, crradicar el agente patógeno cuando sea pertinente e impedir la infección de otros individuos ${ }^{4-8}$. El uso 
de soluciones para hidratación por vía oral (SRO) esta destinado a servir los dos prime ros objetivos. Para que estas soluciones sean eficaces deben ser fáciles de preparar, baratus y seguras para darlas en las condjciones que prevalecen localmente sin riesgo adicional de la salud.

El desarrollo de SRO ha disminuido la mortalidad por alteraciones hidroelectrolíticas asociadas a diarrea aguda ${ }^{9}$. Existe poca información acerca del efecto a largo plazo de distintos esquemas de rehidratación y realimentación sobre la evolución del estado nutricional: la mayoria de las comunicaciones se refiere al corto plazolo. El objerivo de esta investigación fue evaluar las variaciones del estado nutricional que ocurren en niños con diarrea aguda, que habien. do recibido distintos esquemas de tratamicnto son observados durante periodos más prolongados de tiempo.

\section{MATERIAL Y METODOS}

Se estudiaron prospectivamente 45 lactantes menores de 2 años, cuyo grado de deshidratación no era mayor de $5 \%$, que consultaron por diarrea aguda en un Policlínico del Area Sur de Santiago. Desde el punto de vista operacional se definió diarrea aguda como la presencia de 3 u más deposiciones disgregadas o líquidas al dia. Los niños estudiados eran de nivel socioeconómico bajo; su estado nutricional era normal o sufrían de desnutrición leve según los criterios establecidos por Sempé ${ }^{11}$; cl tiempo de evolución antes del ingreso oscilaba entre 24 y 72 horas de duración; no habia recibido antibióticos u otros fámacos an los últimos 15 dias; no sufrían enfermedades intercurrentes que pudieran alterar su estado nutricional o la respuesta del niño a la rehidratación.

Los pacientes fueron asignados a uno de 3 grupos. Los niños del Grupo 1 (G1) recibieron el tratamiento aplicado de rutina en el consultorio. que consistió en suspender las mamaderas pol aproximadamente, 4 a 8 horas desde el momento de la consulta, rehidratar por vía oral con agua con sabor (canela, menta) y azúcar en concentraciones aproximadas a $2 \%$ y ralimentar de manera tal que se alcanzara una ingestión de alimentos comparable a la del mismo lactante antes de la enfermedad, en una semana. De acuerdo con los esquemas usados en el consultorio todos los niños del grupo 1 tecibjeron Furoxona oral, en dosis de acuerdo con el peso. En el grupo 2 (G2) la rehidratación oral de los pacicntes se hizo con una solución comercial (Pedialyte, Abbott) que contiene $30 \mathrm{mEq} \times 1$ de sodio, $20 \mathrm{mEq} \times 1$ de potasio, $4 \mathrm{mEq} \times 1$ de calcio, $4 \mathrm{mEq} \times 1$ de magnesio, $30 \mathrm{mEq} \times 1$ de cloruro, $28 \mathrm{mEq} \times 1$ de lactato y $50 \mathrm{~g} / 1$ de glucosa. La solución se administró "ad libitum", asegurando una inges. tión mínima de $60 \mathrm{cc} x \mathrm{~kg} x$ día, durante los dos primeros dias. En los niños alimentados por lactancia-natural, esta no fue interrumpida; en los que recibian alimentación artificial sc suspendió una mamadera (equivalente a 4 horas de ayuno); luego fueron realimentados con volúmenes $y$ concentraciones crecientes. segun la tolerancia individual, hasta llegar a un aporte de alimentos láctcos y sólidos equivalente a la ingestión previa al episodio de diarrea, en aproximadamente 3 días. Los pacienten del Grupo 3 (G3) recibieron un tratamiento similar al de los niños del G2. excepto que como solución hidratante se empleó la recomendada por la OMS para preparar en el hogar, usando los medios con que cuenta la madre ${ }^{7}$ : I litro de agua, 8 medidas de 5 gramos de azúcar (sacurosa). 3/4 medida de sal y 4 medidas de jugo de limón. En los días 1 y 3 de tratamiento se ubtuvieron alúcuotas de las solu. ciones preparadas por las madres $y$ se midió en clas el contenido de sodio por fotometria de llama. Los niños del G1 y G2 fueron manejados por algunos de los autores, siguiendo el esquema de tratamiento del protocolo de investigación. El día de ingreso al estudio todos los niños fueron examinados: se evaluó su deshidratación, se efectuaron mediciones antropométricas y fucron asignados a un grupo segün orden de llegada. Se obtuvieron muestras de heces para la detección de agentes etiológicos bacterianus, parasitarios $y$ rotavirus. Ese mismo día las madres de los niños de G2 y G3 fueron instruidas respecto a la manera de rehidratar y realimentar al paciente. Posteriormente, todos los lactantes fueron controlados en sus hogares (día 3) y en el consultorio (dia $5,15,25$ y 35). En cada uno de estos controles se evaluó el tratémiento efectuado por ta madre, el estado clínico del niño y se registró su peso y talla. El cstado de nutrición se expresó en relación con el peso para la edad y la talla para la edad. usando como criterios los valores del "National Center for Health Statistics" (NCGS) de los Estados Unidos ${ }^{12}$. Se calculó el porcentaje de adecuación a la mediana para estas dos relacio nes en el momento de la primera consulta, en el día 3 rconsiderando arbitrariamente como el punto en que la hidratación habia llegado a sus valores normales) y en el día 35 (definido arbitrariamente como el punto en que se mediria cl impacto nutricional del episodio de diarrea y de su tratamiento). Se obtuvieron los promedios de los aumentos o disminuciones de peso diarios 
para cada grupo y se compararon con los promedios calculados según las Tablas NCHS para niños sanos de edad y sexo comparables.

La búsqueda de enteropatógenos bacterianos Escherichia coli enteropatogénica (serotipos clásicos), cepas toxigénicas e invasoras, Shigella, Salmonella y Campylobacter, se realizó en una muestra obtenida el día de la incorporación al estudio, mediante una tórula rcctal humedecida y mantenida en medio de transporte de Cary Blair (Culturettc, Marion Scientific, Kansas City, Kansas, EE.UU.). Para el aislamiento de los enteropatógenos se usaron técnicas de rutina. cuyos detalles han sido descritos previamente ${ }^{13}$. La detección de rotavirus se efectuó cn una muestra de aproximadamente 3 gramos de heces, obtenida, durante el primer dia del estudio. El RNA viral se identificó por electroforesis en el gel de acrilamida-bis-acrilamida ${ }^{14}$. Para el estudio pard. sitológico se tomaron tres muestras fecales (una día por mediol que se estudiaron según la técnica de Burrows ${ }^{15 \cdots 16}$.

\section{RESULTADOS}

La investigación incluyó 45 lactantes $(57.8 \%$ vatones) cuyo promedio de cdad fuc $10.7 \pm 5.3$ neses. La duración promedio de la diarrea fue $2 \pm$ 3.9 días. No se demostraron diferencias entre los 3 grupos en la edad de los sujetos, el tiempo de evolución previo al ingreso al estudio y la durjción del cpisodio. El estado de la nutrición de los lactantes al inicio de la investigación fue comparable en los tres grupos, excepto que en $G 3$ hubo 4 nin̄os cuya relación Peso/Edad estaba entre 71 y $80 \%$ de su estándar para la edad, diferencia que fue significativa ( $p<0.005$, Prueba-exacta de Fisher) con respecto a G1 (Tabla 1). No hubo diferencias significativas en la relación Peso/Talla (Tabla 2). Estas tablas muestran además la evolución del $\mathrm{P} / \mathrm{E}$ y $\mathrm{P} / \mathrm{T}$ durante el periodo de observación. La Fig. 1 jlustra las variaciones promedios de peso de cada uno de los grupos en los días controlados. La curva de incremento teórico (percentil 50) se calculó en base a las tablas del NCHS. La comparación del aumento diario promedio de peso observado para cada una de las tres formas del tratamiento al cabo de 35 dias. con los patrones NCHS para sujetos de la misma edad y sexo, reveló que en Gl los niños debian aumentar 13,1 gramos por dia y en realidad aumentaron 12,6 gramosidia; en $\mathrm{C} 2$ los pacientes debían aumentar 12,1 gramos/dia y aumentaron 24,8 y en $G 3$ debían aumentar 11,6 gramosidia $y$ aumentaron 25.8. Las diferencias entre el incremento de peso real y el ideal del grupo $(j l$ no son significativas en ninguno de los intervalos estudiados, incluso al final del estudio. En G2 y G3. en cambio, las dilerencias entre el peso real y el peso idcal fueron significativas desde el día 3 y continuaron aumentando hasta el día 35. Las diferencias observadas entre G1/G2 y G1/G3 entre el inicio y el día 35 son significativas ( $p<0.001$; Prueba de Student). Lus diferencias entre $G 2$ y $G 3$ no fueron significativas en ningún momento de la evolución.

Los esquemas de rehidratación propuestos iueron seguidos estrictamente en todos los casos. Los niños con relación $\mathrm{P}^{\prime} \mathrm{E}$ de liciente se comportaron de manera similar a los bien nutridos. En todos los lactantes la realimentación se llevó a

Tabla 1

Relación Pevo/lidad en 45 lactantes con diarrea aguda al comienzo y ténnino del estudio

\begin{tabular}{|c|c|c|c|c|c|c|c|c|c|c|}
\hline \multirow{4}{*}{$\begin{array}{l}\text { Estado } \\
\text { Nutricional } \\
\text { Peso/Edad * }\end{array}$} & \multicolumn{6}{|c|}{ GRLPOS DE TRATAMILNTO } & \multicolumn{4}{|c|}{ TOTAL } \\
\hline & \multicolumn{2}{|c|}{ Grupo 1} & \multicolumn{2}{|c|}{ Grupo 2} & \multicolumn{2}{|c|}{ Grupo 3} & \multicolumn{2}{|c|}{ Inicial } & \multicolumn{2}{|c|}{ Final } \\
\hline & lnicial & $\overline{\text { Final }}$ & ·ñicial & Final & Inicial & Final & $\mathrm{N}$ & $\%$ & $\bar{N}$ & $\overline{\%}$ \\
\hline & $\mathrm{N}$ & $\mathrm{N}$ & $\mathrm{N}$ & $\mathrm{N}$ & $N$ & $\mathrm{~N}$ & & & & \\
\hline $71 \leqslant 80$ & $0^{* *}$ & 0 & 2 & $\theta$ & $4^{* *}$ & 1 & 6 & 13.3 & 1 & 2.2 \\
\hline $81 \leqslant 90$ & 4 & 5 & 6 & 5 & 4 & 4 & 14 & 31.1 & 14 & 31.I \\
\hline $91 \leqslant 110$ & 11 & 10 & 7 & 8 & 7 & 10 & 25 & 55.6 & 28 & 62.5 \\
\hline $120 \leq 120$ & 0 & 0 & 0 & 2 & 0 & 0 & 0 & 0 & 2 & 4.5 \\
\hline Total & 15 & 15 & 15 & 15 & 15 & 15 & 45 & 100.0 & 45 & 100.0 \\
\hline
\end{tabular}

* PesolEdad: expresado como porcentaje del estándar (NCHS).

** $\mathrm{p}<0.005$ (Prueba exacta de Fisher). 
Tabla 2.

Relación Peso/Talla en 45 lactantes con diarrea aguda. al comienzo y término del estudio

\begin{tabular}{|c|c|c|c|c|c|c|c|c|c|c|}
\hline \multirow{3}{*}{$\begin{array}{l}\text { Estado } \\
\text { Nutricional }\end{array}$} & \multicolumn{6}{|c|}{ GRUPOS DE TRATAMIENYO } & \multicolumn{4}{|c|}{ TOTAL } \\
\hline & \multicolumn{2}{|c|}{ Grupo 1} & \multicolumn{2}{|c|}{ Grupo 2} & \multicolumn{2}{|c|}{ Grupo 3} & \multicolumn{2}{|c|}{ Inicial } & \multicolumn{2}{|c|}{ Final } \\
\hline & $\overline{\text { Inicial }}$ & Final & Inicial & Final & Inicia! & $\overline{\text { Final }}$ & $\overline{\mathbf{N}}$ & $\%$ & $\overline{\mathrm{N}}$ & $\overline{9}$ \\
\hline Pesoitalla (i) & $\mathrm{N}$ & $x$ & $\mathrm{~N}$ & $\mathbf{N}$ & $\mathrm{N}$ & $N$ & & & & \\
\hline $81 \leqslant 90$ & 1 & 1 & 3 & 1 & 3 & 2 & 7 & 15.6 & 4 & 8.8 \\
\hline $91 \leqslant 110$ & 12 & 11 & 10 & 7 & 11 & 8 & 11 & 73.3 & 26 & 57.8 \\
\hline $111 \leqslant 120$ & 2 & 3 & 2 & 5 & 0 & 4 & 4 & 8.9 & 12 & 26.7 \\
\hline$>120$ & 0 & 0 & 0 & 2 & 1 & 1 & 1 & 2.2 & 3 & 6.7 \\
\hline Total & 15 & 15 & 15 & 15 & 15 & 15 & 45 & 100.0 & 45 & 100.0 \\
\hline
\end{tabular}

(1) Peso/Talla: Expresado como \% del estándar (NCHS).

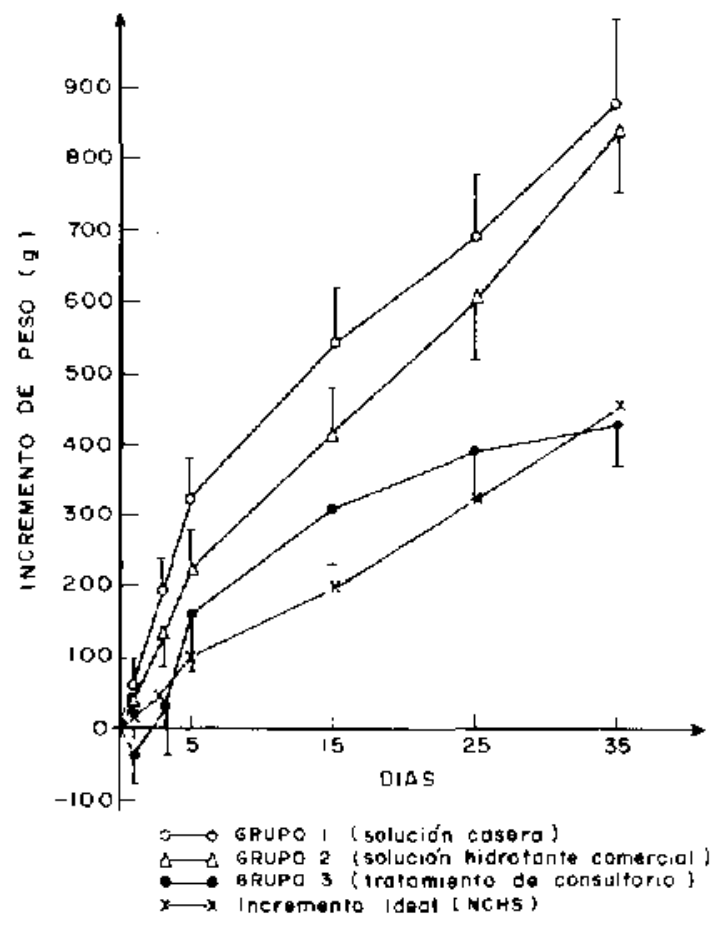

cabo de acuerdo a las indicaciones proporcionadas excepto en 4 pacientes (distribuidos en los 3 grupos), en que esta debió efectuarse con mayor lentitud porque los niños volvieron a presentar deposiciones disgregadas cuando se aumentó la concentración de las mamaderas ( 2 niños), o se introdujeron alimentos sólidos ( 2 niños). En el resto de los lactantes la tolerancia a la realimentación râpida fue adecuada, lo que permitió alcanzar la ingesta láctea y de sólidos habituales para el paciente en aproximadamente 3 dias.

El análisis de las alícuotas de la solución usada en $\mathrm{G} 3$ mostró que el contenido promedio de sodjo fue $62.5 \pm 18.8 \mathrm{mEq} / 1$ (rango $37 \quad 100$ $\mathrm{mEq} / \mathrm{ll}$.

En el $66.6 \%$ de los episodios estudiados se detectó uno o más cnteropatógenos. La lista de agentes bacterianos y virales identificados se muestra en la Tabla 3. La distribución de los agentes identificados por grupo de tratamiento no mostró diferencias de importancia. En Gl se detectaron 9 agentes enteropatógenos bacteria. nos, 2 parasitarios y 4 rotavirus mientras que en G2 estas cifras fueron 6,3 y 3 y en $G 3$ fucron 6,4 y 4 respectivamente

La evolución elínica de los episodios fue igualmente benigna en todos los casos. Ningún lactante requirió rehidratación por via endovenosa ni hospitatización. a pesar de los agentes etiológicos identificados. Ningún niño en G2 $y$ G3 recibio antibióticos.

\section{DISCUSION}

Lá forma en que se efectúan la rehidratación y la realimentación, influye en la evolución clinica de la diarrea aguda. Sin embargo, es difícil medir por scparado sus efectos, sobre todo en plazos largos. Por eso en este estudio no se intentó estudiar en forma separada ambas variables. Se consideró de interés ensayar dos tipos de solu. ciones hidratantes asociadas a un esquema común de realimentación, y comparar estas formas de manejo clinico con el tratamiento que se prescribe actualmente en algunos consultorios.

Las cifras de desnutrición observadas en los niños estudiados fueron mayores que las publicadas por el Ministerio de Salud para menores de 6 años $(8,8 \%)^{17}$. Esto se puede explicar, al menos en parte. porque los estándares de compa- 
Tabla 3 .

Enteropatógenos identificados en 45 lactantes con diarrea aguda

\begin{tabular}{|c|c|c|c|c|c|}
\hline Fistudios & $\begin{array}{c}\text { Casos } \\
\text { Positivos }\end{array}$ & $(9)$ & $\begin{array}{l}\text { Especies } \\
\text { y/o cepas }\end{array}$ & No & $(\%)(\Delta)$ \\
\hline \multirow[t]{7}{*}{ Bacteriológico } & 21 & $(46.6)$ & Campylobacter jejunı & 11 & $(24.4)$ \\
\hline & & & Campylobaster coli & 1 & ( 2.2) \\
\hline & & & Campylobacter ssp & 1 & ( 2.2) \\
\hline & & & ECEP & 6 & $(13.3)$ \\
\hline & & & ECET** & 2 & ( 4.4$)$ \\
\hline & & & ECEI*** & 1 & $(2.2)$ \\
\hline & & & Shigella & 2 & ( 4.4$)$ \\
\hline \multirow[t]{3}{*}{ Parasitológico } & 8 & $(17.7)$ & E. histolytica & 4 & $(8.8)$ \\
\hline & & & Giardia lamblia & 4 & $(8.8)$ \\
\hline & & & A. lumbricoides & 1 & ( 2.2) \\
\hline
\end{tabular}

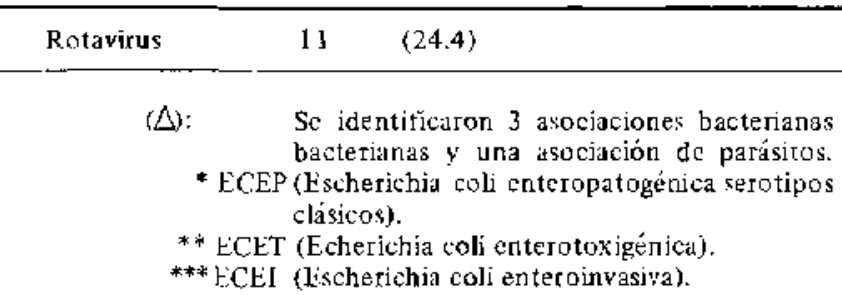

ración usados en este estudio (NCHS), son más exigentes que tos empleados por el Ministerio (Sempé) ${ }^{11}$. Otra explicación posible es que los niños desnutridos tienen mayor susceptibilidad a las infecciones, entre ellas la diarrea aguda, por lo que el grupo estudiado podría representar una muestra scleccionada ${ }^{18-19}$.

El análisis de los incrementos de peso obscrvados en el Grupo 1 indica que el tratamiento, tal como se aplica en el consultorio, repone los volúmenes de agua perdidos por el niño e impide el deterioro del estado nutricional. Sin embargo. la realimentación más temprana y más cnérgica (como las recibidas por $\mathrm{G} 2$ y $\mathrm{G} 3$ ) parece promover la mejoría en plazos más breves.

Podría postularse que por el efecto de la diarrea $y$ de las alteraciones nutricionales que se asocian a ésta. la curva de íncremento de peso debicra estar por debajo del estándar y que la recuperación se produce después de unos días de realimentación. Si se postula que las pérdidas de peso por dcshidratación se cncuentran en todos estos lactantes entre 0 y $5 \%$ y que el peso promedio de ellos era cercano a los $8 \mathrm{~kg}$, la pérdida de agua sería en promedio de aproximadamente $200 \mathrm{~g}$ en cada niño. La rehidratación temprana podría explicar la rápida ganancia de peso que se observa durante los dos primeros díls del estudio. El incremento posterior puede verse acelerado porque algunos de los niños tenian un porcentaje de adecuación Peso'Edad cercano al $90 \%$ del estándar (NCHS). Con el adecuado aporte proteico y energetico administrado durante el estudio, se entraría en un periodo de crecimiento recuperacional ("catch-up growth") que haria que el peso del niño aumente en forma más rápida que lo habitual.

Es dificil explicar la persistencia de la ganarcia de peso de los grupos 2 y 3 hasta 35 dias después del episodio. Sin duda esto es posible en parte porque los niños no desarrollan nuevos episodios de diarrea ni tuvieron otras afecciones significativas durante este periodo. Cabe especular también, que la motivación de las madres de los niños de los yrupos 2 y 3 pueden haber sido mayor, pues las indicaciones del tratamiento fueron dadas por un miembro del equipo utilizando tanto ticmpo como fuera necesario, hasta tener la impresión subjetiva que la madre habia entendido las instrucciones dadas.

Los resultados muestran que la diarrea aguda as tratada efectivamente en el hivel de atención de salud. Sin embargo, la hidratación oral con soluciones que proporcionan combinaciones adecuadas de sodio, potasio y glucosa, sumada a la realimentación precoz, resultan mejores herramientas terapéuticas para evitar el deterioro del estado nutricional del paciente. 
La solución hidratante recomendada por el OMS es una forma de tratamiento seguro, de bajo costo y fácil de aplicar. Su uso precoz podría disminuir la necesidad de hospitalizaciones por diarrea aguda.

Desde el punto de vista etiológico, en ește estudio llama la atención la alta frecuencia con que se aisló Campylobacter $(28,9 \%)$ y la baja incidencia de $E$. coli enterotoxigénica (ECET) (4.4\%). La detección de rotavirus no difiere de las encontradas en otros estudios ${ }^{20}$. En relación a Campylobacter, es difícil explicar los hallazgos observados. Si se considera que los niños estudiados procedian de un área geográfica extensa es improbable que el origen de la infección haya sido un foco comín. Se podría postular que por razones del azar nos hayamos enfrentado a varjaciones naturales relacionadas a la estación, a un brote epidémico, o bien que podrían existir subpoblaciones en que este agente tenga una alta prevalencia ${ }^{21}$. Esta última situación ha sido informada en otros lugares del nundo en desarro$110^{22}$

La baja-incidencia de ECET también es dificil de explicar. Estudios anteriores muestran que on 73 episodios de diarrea pesquisados en una investigación de terreno se aistó este agente en el $23.3 \%$ de los $\operatorname{cosos}^{23}$. En niños hospitalizados por diarrea dguda se ha encontrado en $31.2 \% 24$. Sin embargo, en otro estudio de terreno, en que se siguió una cohorte de niños menores de 1 año, se identificó a ECET en $8.4 \%$ de los episodios ${ }^{25}$. Estos datos sugieren que las incidencias detectadas de ECET podrían variar según los grupos cstudiados de manera semejante a lo descrito para Campylobacter.

El uso de antibióticos durante episodios de diarrea aguda merece un comentario aparte. El criterio internacionalmente aceptado indica que en diarrea aguda simple no se deben prescribir antibióticos, excepto cuando el estudio etiológi. co haya detectado la presencia de Shigella. Si se aplica este criterio a nuestra muestra, la terapia antibiótica habria estado jndicada sólo en el $4.4 \%$ de los casos.

La diarrea continúa representando un problema frecuente en el niño. Las medidas aplicadas por las autoridades de salud han permitido reducir la mortalidad infantil por diarrea agudia a lo largo de los años. La detección temprana de los casos, la rehidratación oral, la realimentación precoz, y el uso de antibióticos cuando estén indicados, son componentes importantes de las actividades tendientes a disminuir la mortalidad causada por esta enfermedad $y$ a interrumpir el ciclo diarrea-desnutrición.

\section{RESUMEN}

Se siguieron durante 35 días tres grupos de pacientes ambulatorios menores de 2 años $(\mathrm{N}=15$ cada uno), que consultaron por diarrea aguda. El Grupo I fue tratado con agua azucarada por vía oral y realimentando de manera que la ingesta previa al episodio se recuperase en una semana. Todos los niños recibieron Furazolidona ora1. E1 Grupo 2 fue rehidratado por vía oral con una solución comercial (Pedialy teM ${ }^{\mathrm{A}}$ ) y realimentado de manera que se recuperara la ingesta habitual en 3 días. Los pacientes del Grupo 3 fueron rehidratados oralmente con una solución preparada en la casa por la madre de acuerdo a recomendaciones de OMS y también volvieron a su ingesta habitual en tres días. Los niños de los Grupos 2 y 3 no recibieron antibióticos. Durante el episodio en estudio $y$ hasta el día 35 de evolución los nîños de los últimos dos gnupos mostraron incrementos promedios diarios de peso significativamente mayores que los del Grupo $1(p<0.001)$. Todos los casos tuvieron un curso clínico benigno y no requirieron rehidratación endovenosa ni hospitalización. El tratamiento efectuado en el Grupo 1 fue efectivo para reponer el agua perdida debido a lá diarrea $\mathrm{c}$ impidió que se deteriorase el estado nutricional de los pacientes. El uso de soluciones hidratantes con proporciones adecuadas de sodio, potasio y glucosa seguido por realimentación precol resultó en mejor recuperación de los parámetros antropométricos nutricionales en menor tiempo.

\section{AGRADECIMIENTOS}

Los autores agradecen a Abbott Laboratories de Chile Ltda., por su contribución en la realización de este proyecto.

\section{REFERENCIAS}

1. Sperotto, G., Carraza, F.R., Marcondes, E.: Treatment of diarthoea dehydration. Am. Clin. Nutr. 30: $1447,1977$.

2. O.M.S.: Manual para el tratamiento de la diarrea aguda. Ginebra, O.M.S., 1980 (Programa de lucha contra las enfemedades diarreicas OMS/CED, SER/8.2).

3. Hirschhorn, N.:The treatment of acute diarhoea in children. An historical and physiological perspective. Am. J. Clin. Nutr. 33:637, 1980.

4. Pizarro, D., Posada, G., Nalin, D.R., Mara, L., Mohs, E.,: Rehidratación por vía oral y su mantenimiento en pacientes de 0 a 3 meses de edad deshidratados por diarrea. Bol. Med. Hosp. Infant. Mex. 37 (5): 879, 1980. 
5. Enfermedades diarreicas. Boletín Epidemiológico (OPS) 3(2): 9, 1982.

6. Duffau, G., Emilfork, M., Calderon, A.: Evałuación de dos fómulas para hidratación oral en el síndrome diarreico agudo con destidratación del lactante. Bol. Med. Hosp. Infant. Mex. 39(11): 729, 1982.

7. La terapia de rehidratación oral (TRO) en el tuatamiento de la Diarrea Infantil. Populat. Rep. Serie L, número 2. octubre, 1981.

8. OPS.: Tratamiento y prevención de la deshidra. tación en las enfermedades diarreicas. Washington, D.C. OMS. 1977. (Publicación Cjentífica No 336).

9. Chen, L.C., Scrimshaw, N.S.: Diarrhoea and malnutrition. Interactions, Mechanisms, and Interactions. New York, N.Y., UNU, 1983.

10. Rohde, J., Cash, R., Guerrant, R., Mahalanabis, D., Molla. A.M. Valyasevi, A.: Therapeutic Interventions in diarthoea. In: Chen, $C$. Scrimshaw, N. Diarrhoea and malnutrition. Interactions, $\mathrm{Me}$ chanisms, and Interventions. New York, UNL, 1983, pp. 287.

11. Sempé, M. Surveillance de la croissence de I'enfant. Reference practiques Le Concours Medicale (París). Supplement No 43, 24 octubre, 1964

12. National Center for health Services. Growth curves for children birth 18 years. United States, Rockville, M.D.: National Center for Health Statistics, 1977, (Vital and Health Statistics. Series 11, No 165, DHEW, publication (PHS) 78-1650).

13. Figuera, G. Troncoso, M., Araya, M., Espinoza, $J$, Brunser, O.: Entetopathogen carriage by healthy individuals living in an area with poor sanitation. $\boldsymbol{J}$. Hyg. Camb. 91:499, 1983.

14. Spencer, E., Arias, M.L.: "In vitio" transeripción catalized by heat treated human rotavirus. J. Virol. 40(1): 1,1981 .

15. Tarres, $P$., Navartere, $N$.: Comparacjón entre los métodos del fijador PAFS y de Teleman modificado en el diagnóstico de protozoos intestinales del hombre. Bol. Chil. Parasitol. 28: 58, 1972
16. Sagua, H., Subrare, V.. Torres. P., Puga, S., Arias, $B$.- Análisis comparativo del rendimiento del fijador PAFS con referencia al método del fijador con alcohol polivinílico, en el diagnóstico de protozoos y Helmintos intestinales. Bol. Chil. Parasitol. 28: 58,1973 .

17. Ministerio de Salud. Anuario de Recursos y Aterciones, 1982. Santiago, Chile, Marzo 1983.

18. Chandra, R.K.: Nutritional deficiency and susceptibility to infection. Bull WHO 57: 167, 1979.

19. Brunser, O., Figueroa, G. Araya, M. Espinoza, $J$. . Infectious and diarthoeal disease. En: Malnutrition. Determinants and consecuences, 10: 259, 1984.

20. Spencer, E., Avendaño, F., Araya, M.: Characteristics and analysis of electropherotypes of human rotavirus isolated in Chile. J. Infect. Dis. 148: 41, 1983.

21. Bokkenheuser, V.D. Richardson, N.J., Bruner, J.H., Roux, J., Shutte, A.B., Koomhof, H.J., Freimen,. , Hortman, E.: Detection of enteric campylobacteriosis in children. J. Clin Microbiol. 9: 227, 1979.

22. Figueroa, G., Troncoso, M., Alcayde, M.P.: Prevalencia de Escherichia coli enteroxigénica en lactantes de nivel socioeconómico bajo. XXI Reunión Anual de la Sociedad Latinoamericana de Investigacon Pediátrica, Montevideo, Lruguay. Noviembre 1983.

23. Aguero, M.E., Reyes, L., Prado, V., Orskov, L., Orskov, F., Cabello, F.: Enterotoxigenic E. oli in a - population of infants with diarrhoea in Chile. J. Clin. Microbiol. 1985 (en prensa).

24. Figueroa, G., Troncoso, M., Araya, M., Brunser, $O$., Espinoza. J., Bustos, M.E.: Role of Campylobacter jejuni in patients with diarthoea and asymptomatic subjects in Santiago, Chile; prospective studies. Third Intemational Workshop on Campylobacter infection. Ottawa, Canada, julio 1985.

25. Espinoza, J., Figueroa, $G_{*}$, Araya $M$. . Spencer, E.. Rowe, B., Hilpert, H., Brunser, O.: Asymptomatic infection and acute diarrhoea in infants. A field study. Fnviado a publicación J. Pediatr. Gastroenterol. Nutr., 1985 . 